\title{
Hyperon production in HIC. The role of the initial state
}

\author{
Genis Musulmanbekov ${ }^{1 \star}$ \\ ${ }^{1}$ JINR, Dubna, Russia
}

\begin{abstract}
A new mechanism is proposed for interpretation of the non-monotonic, enhanced yield of hyperons and positive strange mesons (also known as "horn" effect) that was observed in central heavy ion collisions in the experiment NA49 at SPS. We argue that the data indicate the transition of the nucleons in the overlap region of colliding nuclei with high nuclear density into a certain hyperon phase. This nucleon-to-hyperon transition is accompanied by enhanced yields of positive kaons. The "horn"-effect is a consequence of an interplay between the energy/baryon density attained in the overlap region of colliding nuclei and the overlap time.
\end{abstract}

Understanding the behavior of nuclear matter under extreme conditions of high temperature and density is nowadays a challenge in physics of strong interactions. Many theoretical and experimental efforts have been dedicated to the physics of relativistic heavy-ion collisions looking for signatures of the quark gluon plasma (QGP). Restoration of chiral symmetry and deconfinement are expected to occur at high density and/or temperature of a hadronic matter. In heavy ion collisions even at low and intermediate energies the nuclear density increases more than 2-3 times. The question "How much of incident energy is converted into compression of nuclear matter?" has not found a definite answer yet. Observables indicating non-monotonic and unexpected (from theoretical point of view) behavior of emitted particles is particularly important. In this way the study of the strange particle production in heavy ion collisions is promising as they could serve a good diagnostic tool to investigate the properties of nuclear matter under extreme conditions. Strange particle yields have been thoroughly measured by many experiments with various colliding nuclei at different energies starting from kaon subthreshold production up to energies of SPS and RHIC [1-5]. The systematic study of hadron production in central $\mathrm{Pb}+\mathrm{Pb}$ collisions at SPS performed by NA49 collaboration revealed a sharp structure in energy dependence of positive kaon to pion multiplicity ratio, $K^{+} / \pi^{+}$(Left panel on figure 1 ) [4]. That peculiarity, called "horn"-effect, was later confirmed by Beam Energy Scan (BES) program of STAR collaboration at RHIC [5]. At the same time the ratio of negative mesons $\mathrm{K}^{-} / \pi^{-}$ increases monotonically tending to saturate at higher energies. Traditional transport models were unsuccessful in description of the data. In paper [7] authors claimed non-monotonic dependence of $K^{+} / \pi^{+}$ratio as a signal of the onset of deconfinement. The idea that strangeness is a good signal of deconfinement was put forward by J. Rafelski in 1982 ([13]). The argument was the following: it is energetically favourable to produce $s \bar{s}$ - pairs in deconfined medium than a pairs strange hadrons in hadron gas. The former requires energy $\sim 300 \mathrm{MeV}$, while the strangeness production in hadronic reaction $\mathrm{N}+\mathrm{N} \longrightarrow \mathrm{N}+\mathrm{Y}+\mathrm{K}^{+}$requires $670 \mathrm{MeV}$ above threshold. Albeit a variety of models, statistical

\footnotetext{
^e-mail: genis@jinr.ru
} 

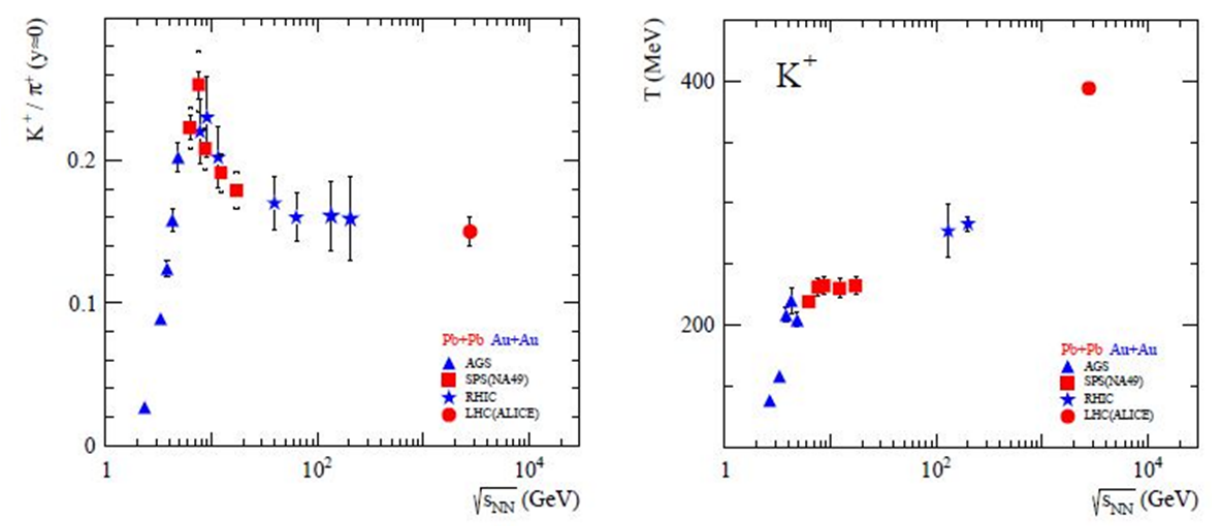

Figure 1. Left panel: $\mathrm{K}^{+} / \pi^{+}$ratio as a function of center of mass energy in central $\mathrm{Au}+\mathrm{Au}$ and $\mathrm{Pb}+\mathrm{Pb}$ collisions. Right panel: Inverse slope parameter of transverse momentum distributions of $K^{+} s$ in these reactions. Data points are from AGS [1-3], SPS [4], RHIC [5] and LHC [6].

[8-10] and kinetic $[11,12]$ (with or without deconfinement) have been proposed for interpretation of "horn" structure a satisfactory understanding of it is still not complete.

We propose a new interpretation of the enhancement of the ratio $K^{+} / \pi^{+}$and the "horn" structure as a consequence of transition of the nuclear matter in a highly compressed zone at the early stage of collision from a nucleonic to hyperonic state. Interpretation of the non-monotonic structure of $K^{+} / \pi^{+}$has initiated intense theoretical activity. Several authors have attempted to reproduce the horn structure employing different approaches. "Horn" - like structure has been predicted in [7], as a manifestation of phase transition between thermalized hadronic and partonic phases. Several versions of Hadron Resonance Gas Model (HRGM) with and without thermal equilibrium describe more or less the effect [8-10]. In some of these models the origin of the horn is related to a transition of the hadron gas from a baryon-dominated to a meson-dominated gas at $\sqrt{ } s_{N N} \approx 8 \mathrm{GeV}$ along the freezeout curve. Authors in [12] elaborated the hadronic kinetic model in which the interplay between the available energy density and the lifetime of the fireball was able to describe data under the assumption that the total lifespan of the fireball is decreasing function of the collision energy for SPS energies above $30 \mathrm{AGeV}$.

Here we propose a new interpretation of non-monotonic behavior of $K^{+} / \pi^{+}$ratio in heavy ion central collisions. At the initial phase of head-on collision the nuclear density $\rho$ in the overlap/collision zone can reach several times the nuclear saturation density $\rho_{0}$. Since nucleons are extended objects of dimension $\sim 1 \mathrm{fm}$ each nucleon undergoes compression and occupies more than two times smaller volume than what was typical at the saturation density. Obviously, nucleons under such a strong compression/pressure have to modify their properties. We assume that under such conditions it is more preferable to nucleons to transit into a hyperonic state since hyperons containing one, two or three strange quarks occupy smaller volume than nucleons composed of light quarks. Thus, at the early stage of collision, before creation of a hot and dense fireball, a condensate of $s \bar{s}$ - pairs is created in the compressed overlap zone. Then $s$-quarks replace one (two, three) $u$ or $d$ quarks of nucleons, and $\bar{s}$ - quarks form with those replaced quarks strange mesons: $p, n \longrightarrow$ hyperons + kaons. Therefore, in this "action-reaction" mechanism the transition of nucleons to hyperons is realized through the following channels: 


$$
\begin{aligned}
p(u u d) & \longrightarrow \Sigma^{+}(u u s)+K^{0}(d \bar{s}) \\
& \longrightarrow \Sigma^{0}(u d s)+K^{+}(u \bar{s}) \\
& \longrightarrow \Lambda^{0}(u d s)+K^{+}(u \bar{s}) \\
& \longrightarrow \Xi^{-}(d s s)+2 K^{+}(u \bar{s}) \\
& \longrightarrow \Xi^{0}(u s s)+K^{+}(u \bar{s})+K^{0}(d \bar{s}), \\
& \longrightarrow \Omega^{-}(s s s)+2 K^{+}(u \bar{s})+K^{0}(d \bar{s}),
\end{aligned}
$$

$$
\begin{aligned}
n(u d d) & \longrightarrow \Sigma^{-}(d d s)+K^{+}(u \bar{s}) \\
& \longrightarrow \Sigma^{0}(u d s)+K^{0}(d \bar{s}) \\
& \longrightarrow \Lambda^{0}(u d s)+K^{0}(d \bar{s}) \\
& \longrightarrow \Xi^{0}(u s s)+2 K^{0}(d \bar{s}) \\
& \longrightarrow \Xi^{-}(d s s)+K^{+}(u \bar{s})+K^{0}(d \bar{s}) \\
& \longrightarrow \Omega^{-}(s s s)+2 K^{0}(d \bar{s})+K^{+}(u \bar{s})
\end{aligned}
$$

According to the transition channels the $K^{+} \mathrm{s}$ and $K^{0} \mathrm{~s}$, but any $K^{-}$are produced only. $K^{-}$can be produced in reactions $\pi Y \longrightarrow N K^{-}$and $\phi \longrightarrow K^{+} K^{-}$. The first reaction should be suppressed because pions, composed of light quarks do not survive in a dense hadronic matter. $\phi$-meson production in hadronic reactions are suppressed by OZI-rule. However, formation of a condensate of strange quarks in a dense matter can lead to weakening of OZI-rule, and the yield of $K^{-} \mathrm{s}$ at energies below "horn"effect is due to $\phi$-mesons decay. If this "action-reaction" mechanism is pertinent for the description of the "horn" structure, then the ratio $K^{0} / \pi^{0}$ should behave in the same way.

Another contribution to strange particle production comes from the kinetic mechanism of binary reactions. At low and intermediate energies the $K^{+} \mathrm{s}$ are produced predominantly in so-called "associated" reactions $N+N \longrightarrow N+Y+K^{+}, \pi+N \longrightarrow Y+K^{+}$where $N$ stands for nucleons and $Y$ $(\Lambda, \Sigma, \Xi, \Omega)-$ for hyperons. $K^{-}$s can be produced on the next step, in reaction $\pi+Y \longrightarrow N+K^{-}$. As the collision energy increases, the contribution of these reactions to inelastic cross section decreases, due to increasing phase space that leads to increasing number of channels of strange particle production. Hence, one could argue that this (associated) production mechanism is sufficient to be consistent with horn-like non-monotonic $\mathrm{K}^{+}$spectrum. However, the results of transport models, URQMD and HSD, demonstrated that this mechanism was not able to describe quantitatively experimental data [14].

There are two factors providing the proposed transition mechanism: compression energy deposited into collision zone and time during which two colliding nuclei are overlapped - overlap time. Both are functions of collision energy. Which part of available energy is converted in creation/condensation of $s \bar{s}$ - pairs with subsequent formation of hyperons and kaons? This question addresses to compression of nuclear matter which could be derived from equation of state (EOS), relation between an energy density and baryonic density in the interaction zone. However, extraction of information on EOS both from experiment and theory is a matter of long debate. At high nuclear densities there are many uncertainties that make it difficult to extract the compression from experimental data. There are two hydrodynamical scenarios of initial conditions in hadron or heavy-ion collisions: Landau solution and Bjorken solution. In the Landau solution a compression in collision zone should be maximal because of the fully stopping power of hadronic matter. In contrast to the Landau scenario the core assumption of the Bjorken scenario is the boost-invariance that gives an accelerationless solution (zero stopping power) of the hydrodynamical equations. Truth - somewhere in the middle. For qualitative considerations we assume that the available energy to be converted into nuclear compression, $E_{\text {comp }}$, in the overlap zone is an increasing function of baryonic density.

On the other hand, the overlap time, $\tau_{o}$, is decreasing function of the collision energy. Transition of nucleons to hyperons accompanied by strange meson production should take finite time which we denote by transition time, $\tau_{t r}$. Naturally to expect that $\tau_{t r}$ is order of the nucleon size, $\approx 1 \mathrm{fm}$. Hence the proposed mechanism of transition can be realized if this transition time, $\tau_{t r}$, is less than the overlap time, $\tau_{o}$, when the net baryon density is maximal (on the average) at a given collision energy. 

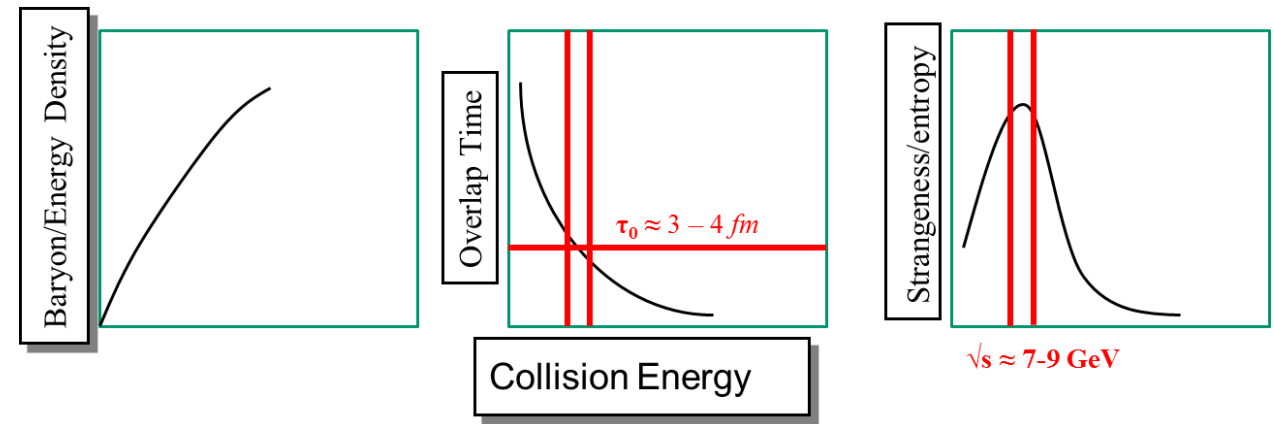

Figure 2. Interplay of compression energy increasing with collision energy (left panel) and overlap time decreasing with collision energy (middle panel) gives a peak of $K^{+} / \pi^{+}$(right panel). Vertical lines outline location of the peak, and the horizontal line depicts the corresponding overlap time for gold-gold collisions.

Thus, the collision energy dependence of the probability of nucleon-to-hyperon transition is defined by interplay of the increasing compression energy and decreasing overlap time:

$$
P_{N \rightarrow Y} \propto\left(\tau_{o} / \tau_{t r}\right)^{\alpha} E\left(\rho / \rho_{o}\right)_{c o m p}
$$

where $\alpha$ is the adjustable parameter and $\rho_{0}$ is the nuclear saturation density. This interplay results in the non-monotonic behaviour of yields of hyperons and $K^{+}$s. Besides energy dependence the overlap time depends on the sizes of colliding nuclei. For symmetrical nuclei in the center of mass system $\tau_{o}=\left[2 R_{A} /(\gamma \beta)\right] b_{S P}$, where nuclear radii, $R_{A}=1.2 A^{1 / 3}, \gamma-$ gamma-factor and $\beta$ - velocity of colliding nuclei. Factor $b_{S P}$ stands for taking into account the effect of stopping power. Following the Bjorken scenario we set the minimal value for the stopping power $\left(b_{S P} \sim 1\right)$. Then the peak of $K^{+} / \pi^{+}$for gold-gold collisions according to the data taking place at $\sqrt{ } s=7-9 \mathrm{GeV}$ corresponds to $\tau_{o}=3-4$ fm (figure 2).

Additionally to the non-monotonic behaviour of the ratio $K^{+} / \pi^{+}$the experiments observed at the same energies, so-called, "step" structure, the plateau in the inverse slope of transverse momentum distributions both of $K^{+}$and $K^{-}$(Right panel on figure 1). According to our scenario this plateau emerges when kaon production via mechanism of binary interactions starts to dominate over the nucleon-to-hyperon transition mechanism, inasmuch as transverse momenta of kaons produced in binary interactions should be softer than those ones in transition mechanism. Up to the "horn" peak the $K^{-}$-mesons should be produced mainly in decays of $\phi$-mesons which are created in the strange quark condensate, violating OZI suppression rule. Obviously, their transverse momentum spectra should be harder than those ones of kaons produced in binary interactions. These considerations are supported by observed in experiments strong enhancement of $\phi$-mesons yield near and above threshold [15-17]. Also, compression of the nuclear matter in the overlap volume can lead to domination of vector meson production ( $\rho$ and $\omega$ ) over pseudoscalar ones (pions). The enhanced yield of dileptons with invariant masses near the masses of vector mesons in comparison with the spectrum from elementary nucleon-nucleon reactions was observed by DLS and HADES [18, 19].

\section{References}

[1] L. Ahle et al., Phys. Lett. B 490, 53 (2000)

[2] J. L. Klay et al., Phys. Rev. Lett. 88, 102301 (2002) 
[3] L. Ahle et al., Phys. Rev. C 60, 064901 (1999)

[4] S. V. Afanasiev et al., Phys.Rev. C 66, 054902 (2002). C. Alt et al., Phys. Rev. C 77, 024903 (2008)

[5] L. Kumar (STAR Collaboration), J. Phys. G:Nucl. Part. Phys. 38, 124145 (2011)

[6] M. Floris (ALICE Collaboration), J. Phys. G:Nucl. Part. Phys. 38, 124025 (2011)

[7] M. Gazdzicki and M. Gorenstein, Acta. Phys. Pol. B 30, 2705 (1999)

[8] J. Cleymans, H. Oeschler, K. Redlich, and S. Wheaton, Phys.Lett. B 615, 50 (2005)

[9] A. Andronic, P. Braun-Munzinger and J. Stachel, Nucl.Phys. A 834, 237C (2010)

[10] K.A. Bugaev et al., arXiv:1412.6571v2 (2014)

[11] J.K. Nayak et al., Acta Phys. Slov. 56, 27 (2005)

[12] B. Tomasik and E.E. Kolomeitsev, Eur. Phys. J. C 49, 115 (2007)

[13] J. Rafelski, Phys. Rep. 88, 331 (1982)

[14] E. Bratkovskaya et al., Phys. Rev. C 69, 054907 (2005)

[15] B. B. Back et al., Phys. Rev. C 69, 054901 (2004)

[16] C. Alt et al., Phys. Rev. C 78, 044907 (2008)

[17] G. Agakishiev et al., Phys. Rev. C 80, 025209 (2009)

[18] R. G. Porter et al., Phys. Rev. Lett. 79, 1229 (1997)

[19] A. Rustamov et al., Int. Jour. Mod. Phys. A 26, 384 (2011) 